\title{
Optical constants of powdered limestone obtained by taking into account the grain shapes: Applicability to Martian studies
}

\author{
A. Jurewicz ${ }^{1}$, V. Orofino ${ }^{2}$, A. C. Marra $^{2}$, and A. Blanco ${ }^{2}$ \\ 1 Space Research Centre, Polish Academy of Sciences, Bartycka 18a, 00-716 Warsaw, Poland \\ e-mail: aaj@cbk.waw.pl \\ 2 Department of Physics, University of Lecce, Via Arnesano, CP 193, 73100 Lecce, Italy \\ e-mail: anna.cinzia.marra@le.infn.it; blanco@le.infn.it
}

Received 4 February 2003 / Accepted 14 August 2003

\begin{abstract}
The modelling and the interpretation of infrared spectra exhibited by astronomical dusty objects require fair acquaintance with complex refractive indices, the so-called "optical constants", of cosmic analog materials. It turns out that the spectra of the latter, in case of a crystalline granular material, depend on the size and the shape of the grains and may differ from the spectra of the same material but in bulk form. This phenomenon can be very elegantly accounted for by considering optical lattice excitations specific to small particles, the so-called "surface modes". We present a study of the spectral behaviour, in the 1.5-62.5 $\mu \mathrm{m}$ range, of the optical constants of a particulate sample of limestone, a typical carbonate material mainly composed of calcite $\left(\mathrm{CaCO}_{3}\right)$. Shape effects have been accounted for by considering a collection of randomly oriented ellipsoids with various continuous distributions of shape parameters. It is shown that in the spectral region around the bands at $32 \mu \mathrm{m}$ and $44 \mu \mathrm{m}$, whose assignment to surface modes raises no doubt, the optical constants derived for various shape distributions are markedly different from each other. We find that the best agreement between laboratory and theoretical spectra is obtained for spheres while for two continuous distributions of ellipsoids the fits are slightly worse. In other words the optical constants, that describe best the interaction between electromagnetic radiation and our limestone sample, are those derived by using Mie theory (valid for spheres); this is in agreement with SEM analysis which indicates a spheroidal shape of the particles.

Such conclusions, valid for limestone particles, cannot be extrapolated directly to other particles and/or materials, since every case has to be treated independently. They should nevertheless be helpful in avoiding the possible error of interpreting absorption spectra of particulate crystalline stuffs without taking into account the effects of particle size and shape.
\end{abstract}

Key words. methods: numerical - planets and satellites: individual: Mars

\section{Introduction}

In spite of a great amount of information recently received from several space missions (Golombek et al. 1997; Albee et al. 2001), there are still unanswered questions about the climatic evolution of Mars (Haberle 1998; Carr 1999) and its connection with the possible development of some form of life on the planet (Mc Kay et al. 1996; Thomas-Keprta et al. 2000).

Many indications seem to suggest that the Martian climate has not always been so harsh as it is today and that in the past the surface temperature and atmospheric pressure allowed the stability of liquid water on the surface of the Red Planet. This hypothesis, supported by climatic models (Pollack et al. 1987; Forget \& Pierrehumbert 1997; Yung et al. 1997) and morphological studies of the surface of Mars (Masursky et al. 1977; Malin \& Carr 1999), has been strengthened by the discovery, in the Martian meteorites, of weathering deposits produced by liquid water filling the cracks and voids in the rock

Send offprint requests to: V. Orofino, e-mail: orofino@le.infn.it within the last billion years (Sawyer et al. 2000). According to this hypothesis, on the surface of Mars, in the past, large bodies of standing water, very similar to terrestrial lakes or seas, could be present (Baker et al. 1991; Scott et al. 1995; Cabrol \& Grin 1999). In these aqueous environments carbonate deposits should have been produced. The identification of such deposits, which could have survived in some regions of the planet, should indicate that the ancient climate of Mars was very different from the present one. For this reason the search for carbonate deposits on Mars is a very important topic in the study of the paleoclimate of the Red Planet.

On the other hand, because of strong winds blowing on the surface, it is reasonable to search for carbonate compounds also in the atmospheric dust. Despite extensive work on this subject, however, no evidences about the spectroscopic detection of carbonates on the surface and in the atmosphere of Mars have been found, so that the question is still open (Fonti et al. 2001). The possible presence and detectability of coated particles produced by the photodecomposition of the outer layer 
of carbonate grains have been discussed in a previous paper (Orofino et al. 2000). According to the results of this work, the photodecomposition of carbonates, if possible on Mars at all (Jurewicz et al. 2001), should not prevent spectroscopic detection of these minerals on the surface and in the aerosol of the Red Planet, and more likely at particular sites on its surface.

In this context a fairly precise knowledge of the frequency dependent complex refractive index ("complex optical constants") of a typical carbonate, such as limestone, may help to elucidate the problem of the presence of this kind of minerals on Mars. Limestone, in fact, is a carbonate-bearing material mainly composed of calcium carbonate $\left(\mathrm{CaCO}_{3}\right)$, generally in the form of very small calcite grains. For the purpose of modelling the synthetic spectra of Martian atmospheric dust, to be compared with observations, the optical constants measured for bulk stuff have been generally used until now. It is however important to note that, when bulk optical constants of various materials are used in theoretical calculations, the agreement between laboratory and computed spectra can be very poor (Huffman 1988). On the contrary, the experimental extinction spectra of submicron particles can be well reproduced by using optical constants directly obtained in the laboratory for particulate materials. Caution must therefore be exercised in extrapolating laboratory results concerning bulk samples, to physical conditions where particles smaller than the wavelength of the radiation are expected to occur. The disagreement has genuine physical roots, and in Sect. 2 we outline an explanation of the issue. In fact, the incoming radiation can induce lattice vibrations in the particulate material, the so-called "surface modes", specific to the shape and size of the grains. In a first attempt infrared (IR) transmission spectra measured for particles with an average radius of $0.06 \mu \mathrm{m}$, were used in previous works to retrieve optical constants of submicron grains (Orofino et al. 1998, 2002). In these cases the dispersion theory, under the assumption of the spherical shape of the grains and hence according to Mie theory, was applied to this purpose. The data were also used for a tentative identification of carbonates on Mars. However, a Scanning Electron Microscopy (SEM) analysis of these limestone grains has shown that the particle shapes are different from spheres. As a matter of fact the real grains, whether on Mars or in laboratory samples, in general are not spherical and for this reason Mie theory can be used in principle only as an approximation for the derivation of the optical constants.

In the present paper we want to examine whether Mie approximation is sufficient to derive particulate optical constants able to reproduce the spectra of small real limestone particles, or the effects of particle shape may be better mimicked by particulate optical constants obtained considering a collection of randomly oriented ellipsoids with various continuous distributions of shape parameters (Rouleau \& Martin 1991; Zubko et al. 1996). Two shape distributions, a flat continuous distribution of ellipsoids (CDE) with equal single volumes (Bohren \& Huffman 1983), and a modified CDE, will be discussed in more detail in Sect. 3. In Sect. 4 we present and discuss the results of the best fits to the experimental spectra by comparing them with previous data.

\section{Bulk optical constants versus particulate optical constants}

There exists convincing experimental evidence that characteristic spectral structures which show up in the IR reflection or transmission spectra of a crystalline material, depend on whether the studied sample appears in bulk or particulate form (Conel 1969; Hunt \& Logan 1972; Huffman 1977). Indeed, in addition to the frequency dependent complex refractive index proper for the bulk stuff, also the size and shape of the grains can be singled out among the factors which affect the aspect of a particulate spectrum (Hunt \& Logan 1972). The bulk refractive index is well defined in the realm of electromagnetic waves described by Maxwell equations, and in principle can be easily related to the optical properties, e.g., of a large crystalline slab. When, however, granular crystalline samples are studied, this is in general not true, in spite of several, often successful attempts to describe the optical properties of particulate materials in the IR. Refractive indices derived for bulk materials prove often inadequate when used to explain the interaction of IR radiation with granular samples of composition identical to bulk parent stuff, especially when particles are small compared to the wavelength of radiation (Huffman 1988). A legitimate doubt may then be raised whether the concept of a unique, frequency dependent complex refractive index giving full account of the optical properties of a crystalline stuff, irrespectively of its physical form, is acceptable indeed.

In the following, an attempt to clarify this issue is undertaken. Actually it turns out that, under some limitations pertinent to the size of the grains, the phenomena pointed out in the foregoing have since long been very elegantly accounted for within the framework of the purely classical theory of optical lattice vibrations-optical phonons (Huang 1951; Born \& Huang 1954; Englman \& Ruppin 1968; Ruppin \& Englman 1968; Ruppin \& Englman 1970). To render the issue clear, the concepts and conclusions of the theory relevant to the aims of this paper are discussed here for the case of simple diatomic, single-mode ionic crystals as e.g. $\mathrm{NaCl}$. Generalisation to complex systems, however, does not alter the main conclusion "that a theory which does not take into account the size and shape of the specimen can only partially explain absorption and emission results"(Englman \& Ruppin 1968).

Under consideration are then infinite diatomic crystals with optical isotropy, satisfying the so-called periodic boundary conditions, meaning that each ion is considered to be equivalent to every other ion of the same species. The macroscopic equations for phonons of wavelength large compared with the lattice constant, when considered in conjunction with Maxwell equations (i.e. including retardation but with attenuation neglected), allow in this case for three types of vibrational solutions for the relative displacement of the ions $\boldsymbol{f}(\boldsymbol{r})$ in the coupled phononphoton (so-called polariton) system:

1) longitudinal modes with real frequency $\omega=\omega_{\mathrm{L}}$, the socalled longitudinal optical frequency of the infinite crystal;

2) transverse modes with real frequencies $\omega>\omega_{\mathrm{L}}$ or $\omega<\omega_{\mathrm{T}}$, where $\omega_{\mathrm{T}}$ is the so-called transverse optical frequency of 
the infinite crystal, such as the condition $\omega_{\mathrm{L}}>\omega_{\mathrm{T}}$ holds (Lyddane et al. 1941);

3) modes of different type, later named surface modes, with real frequencies $\omega_{\mathrm{T}}<\omega<\omega_{\mathrm{L}}$.

In the infinite crystals under discussion the normal modes of the system are plane waves. This follows from the solution of the Helmholtz equation for $\boldsymbol{f}(\boldsymbol{r})$ and applies to the modes of type 1 and 2 . The essential point about type 3 solutions is that the Helmholtz equation allows then only for solutions with spatial exponential dependence. Therefore in the case of an infinite lattice with periodic boundary conditions, type 3 solutions regular everywhere in the crystal do not appear at all. For finite specimens however, when conditions on their confining surfaces have to be imposed on $\boldsymbol{f}(\boldsymbol{r})$, way is given to non-trivial results. The presence of a boundary, in fact, breaks the translational symmetry of the crystal what finally leads to the appearance of physically meaningful type 3 solutions - the surface modes. Their number is roughly proportional to the number of surface cells in the crystal. Consequently the polariton eigenfunctions and frequency eigenvalues, and in turn characteristic features of the IR spectrum, become dependent on the shape and size of the crystal. When damping is taken into account, the frequencies corresponding to these vibrational modes turn out complex but close to them at real (physical) frequencies an enhancement in absorption or, equivalently, a resonance in absorption shows up (Huffman 1977).

There exist several thorough discussions of bulk and surface transverse modes that develop in crystal samples of various shapes. One approach used to this purpose has been the so-called dielectric continuum theory where the grain material is characterised by a bulk, frequency dependent dielectric function $\epsilon(\omega)$ (Englman \& Ruppin 1966; Fuchs et al. 1966; Fuchs \& Kliewer 1968; Fuchs 1975, 1987). Formally, the task of finding out the surface modes consisted here in solving the Helmholtz equation with appropriate boundary conditions for the field, imposed on some surfaces representing the grain shapes. In the other approach, based on lattice dynamical calculations, a finite crystal is considered to be a large molecule. In this way the atomicity of the medium is taken into account (Genzel \& Martin 1972a,b; Martin 1975; Chen et al. 1978). A comparison of the two types of calculations was carried on for a thin crystal slab (Fuchs 1973) and for cubic microcrystals (Fuchs 1978). The relevance of this comparison to our study of surface modes will be discussed later in this section.

As a particular case of a dielectric continuum theory for spherical grains of radius $r$, Mie theory was shown (Ruppin \& Englman 1970) to give full account of the boundary conditions and to describe properly both the retardation and the surface modes. The eigenfrequencies $\omega_{q}$ of the system are then given by the expression

$\omega_{q}^{2}=\omega_{\mathrm{T}}^{2} \frac{\epsilon_{0}+\epsilon_{\mathrm{M}}(q+1) / q}{\epsilon_{\infty}+\epsilon_{\mathrm{M}}(q+1) / q}, \quad q=1,2,3, \ldots$

where $\epsilon_{0}$ denotes the dielectric constant for static fields, while $\epsilon_{\infty}$ is the dielectric constant for frequencies high above $\omega_{\mathrm{L}}$, but below the frequencies of electronic transitions. The frequencies $\omega_{q}$ do not depend on the radius, but the amplitude of vibration decreases here as $r^{q-1}$ with increasing distance from the surface what justifies the naming surface modes.

The answer to the doubt expressed at the beginning leads then to the important conclusion that the concept of a frequency dependent complex refractive index for a given material makes sense only if the expression for $\epsilon(\omega)$ proper for a sufficiently large ("infinite") bulk stuff is completed with terms following from the boundary conditions relevant to a given sample.

Some remarks to the formulation of this concept are due. The mathematical complexity of the general theory of the Helmholtz equation (Morse \& Feshbach 1953) makes the concept effectively applicable only to a very limited class of simplest forms of the grains. Yet, nothing prevents using it as a basic conceptual guide in interpreting the spectra of crystalline samples. Furthermore, the relevance of the possible surface corrections to the shaping of the spectra for particulates may be difficult to be assessed a priori, but certainly depends on the chemical and physical properties of the grains. It is known, in fact, that bulk optical constants for e.g. palagonite (Roush et al. 1991) reproduce properly the features of the spectra for granular specimens (Muci et al. 1997).

We go back now to small grains, such that the characteristic IR radiation wavelength is comparable to, or larger than, the particle size. In this case retardation is negligible, and the Rayleigh approximation based on the equations of electrostatics gives full account of the interaction of electromagnetic radiation with the lattice. Only the lowest surface modes are then important. In the case of spheres this is the Fröhlich mode (Fröhlich 1949), obtained from Eq. (1) with $q=1$ :

$\omega_{F}^{2}=\omega_{T}^{2} \frac{\left(\epsilon_{0}+2 \epsilon_{M}\right)}{\left(\epsilon_{\infty}+2 \epsilon_{M}\right)}$.

For tri-axial ellipsoids the lowest surface modes corresponding to vibrations along the $i$ th principal semi-axis have a frequency given by (Grindlay 1965):

$\omega_{1 i}^{2}=\omega_{\mathrm{T}}^{2} \frac{\epsilon_{0}+\epsilon_{\mathrm{M}}\left(1 / L_{i}-1\right)}{\epsilon_{\infty}+\epsilon_{\mathrm{M}}\left(1 / L_{i}-1\right)}$,

where $L_{i}$ is a geometrical factor depending on the relative lengths of the three main semi-axes (Bohren \& Huffman 1983).

A comparison of the eigenfrequencies as given by Eqs. (2) and (3) evidences the potential relevance of the grain shape in the studied medium on its spectrum in the IR.

An important point to be considered is the grain size at which surface modes become dominant in experimentally measurable quantities, such as e.g. the spectral absorption coefficient. In laboratory work with granular samples, well known is the apparent "shift" of absorption peaks from their positions for bulk material to frequencies higher than $\omega_{\mathrm{T}}$. The effect appears at $\omega_{\mathrm{T}} d / c \sim 1$ where $d$ denotes the characteristic dimension of the grain (Bryksin et al. 1974). Our earlier considerations make it clear that in fact, this is not a "shift" but surface modes begin to be detected instead of bulk modes. The latter always have frequencies below $\omega_{\mathrm{T}}$, and are insensitive to the dielectric properties of the surrounding medium. As the numbers of surface and bulk polaritons are approximately proportional to the number of surface cells, and to the whole of cells in 
the crystal, respectively, the ratio of surface to bulk absorption increases with shrinking crystal size. According to Bohren \& Huffman (1983) surface modes at wavelength $\lambda$ are dominant at grain sizes satisfying the condition $\pi d / \lambda<0.1$ which constraints the upper limit for the sizes by $\omega_{\mathrm{T}} d / c<0.2$. The case of $\mathrm{NaCl}$, the crystal used already here as an example, might in this respect be quite instructive. Bryksin et al. (1971) report an experimental study on the development of surface modes with decreasing grain size of particulate $\mathrm{NaCl}$ samples in the far IR transmission spectrum. In this case $\omega_{\mathrm{T}} / c=0.10 \mu \mathrm{m}^{-1}$ what corresponds to the wavelength $\lambda_{\mathrm{T}}=61.1 \mu \mathrm{m}$. At $d \sim$ $30 \mu \mathrm{m}$, and $\omega_{\mathrm{T}} d / c \sim 3$ a broad absorption band spans over the bulk and surface frequency ranges from well below $\omega_{\mathrm{T}}$ up to $\omega_{\mathrm{L}}$. With decreasing grain sizes the band narrows and becomes concentrated in the range $\left[\omega_{\mathrm{T}}, \omega_{\mathrm{L}}\right]$ when $d \lesssim 2 \mu \mathrm{m}$ corresponding indeed to $\omega_{\mathrm{T}} d / c \lesssim 0.2$, in agreement with the criterion of Bohren \& Huffman (1983). In calcite crystals, the fundamental mode of interest to us has $\omega_{\mathrm{T}} / c=0.098 \mu \mathrm{m}^{-1}$ (Landolt-Börnstein 1955), so that the same criterion is setting again $d \lesssim 2 \mu \mathrm{m}$ as the particle size when surface modes are becoming fully effective in the IR spectrum.

Another very interesting issue concerns the applicability of the continuum theory to the grain size as discussed above. The studies of Fuchs $(1973,1978)$ mentioned before, show that optical absorption by microcrystals of size corresponding even to $\sim 10$ atomic layers $(\sim 0.002 \mu \mathrm{m})$ obtained independently from continuum and lattice dynamics calculations, still are in qualitative agreement! The discrepancies take the form of excess broadening of the resonance peaks in continuum calculations. It was shown by Duley (1976) that the effect could be caused by the existence of surface stresses, because the fraction of surface cells in the particle is large, and changes in the forces acting on the atoms become important. The continuum theory becomes inadequate if the characteristic size of the particles is comparable to the interatomic distance, typically less than $0.0005 \mu \mathrm{m}$. At this stage the description of the material by means of a bulk optical constant (with additional boundary conditions) is not possible any more. Simultaneously it appears (Genzel \& Martin $1972 b)$ that calculated lattice vibrations of microcrystals containing only 64 atoms ( $\sim 4$ atoms along the edge) closely approximate the lattice vibrations of a crystal when cyclic boundary conditions are assumed, and also those measured in experiments. This is really surprising if one realises that 56 of the whole 64 atoms are on the surface. Classical lattice calculations ensure therefore smooth transition from large macroscopic samples to minute microcrystals.

In the light of the preceding remarks, the object of the present study, namely the calculation of complex optical constants in the IR for grains of limestone with non-uniform shapes, certainly does not represent a task that could be solved by strict application of the outlined analytic procedures. This is essentially because of the difficulty with accounting for the complicated boundary conditions on the surface of the real grains. For this reason we decided to use a semi-empirical approach which implicitly takes into account the boundary conditions on the surface of the grains. Our procedure consists in fitting the experimental spectra by using Lorentz dispersion

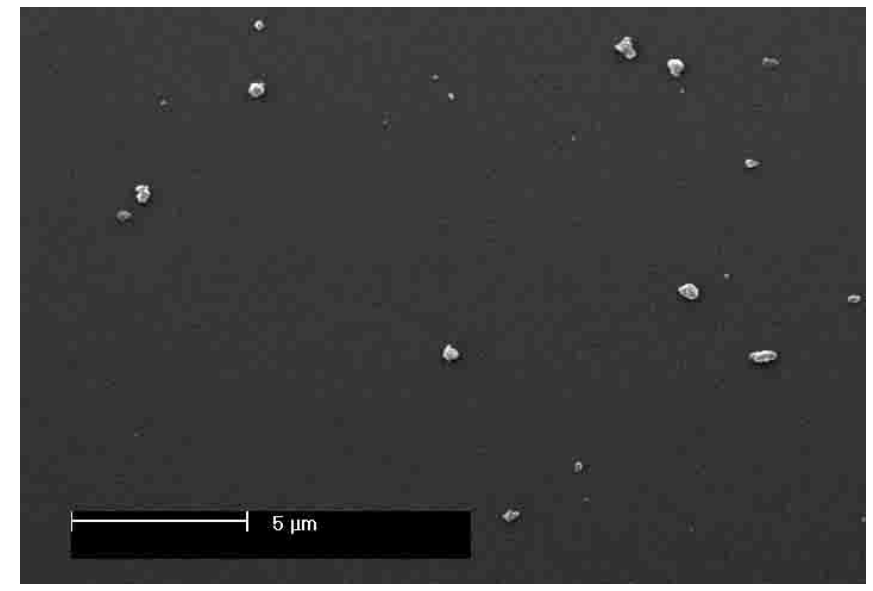

Fig. 1. SEM image of our limestone sample taken by means of a Philips XL 20 electron microscope. The mean size of the particles is $0.12 \mu \mathrm{m}$.

theory in order to directly obtain particulate optical constants. We are going to discuss this procedure in the following section.

\section{Optical constants retrieving procedure}

The procedure of retrieving the optical constants for powdered limestone presented here is similar to the approach followed by Long et al. (1993) and Orofino et al. (1998, 2002). In the present case, however, it becomes important to have the whole procedure subjected to the main conclusion outlined in Sect. 2. The SEM analysis of our limestone grains indicates that the particles have an average size of $0.12 \mu \mathrm{m}$. In other words our particles have size greater than the above-quoted limit $(0.0005 \mu \mathrm{m})$ for the applicability of the continuum theory and, at the same time, they are small enough to allow the presence of surface effects. This means that the correct reproduction of the spectral structures belonging to surface modes in the fitting procedure is expected to be sensitive to the shape of the particles. In particular SEM images (see Fig. 1) show that, though nearly spheroidal, the grains are not perfectly spherical. For this reason it is necessary to examine whether Mie approximation is sufficient to reproduce the spectra of small real limestone particles, or the effects of particle shape may be better mimicked by considering a collection of randomly oriented ellipsoids with various continuous distributions of shape parameters (Rouleau \& Martin 1991; Zubko et al. 1996).

The interest in the possibilities offered by a collection of such forms has a two-fold motivation. For the first, a distribution of ellipsoids comprising a variety of forms from needles to spheres, to thin disks, offers a chance to approximate fairly well the real shapes in a collection of particles. For the second, an ellipsoid allows for a formally exact treatment in the framework of the Rayleigh approximation. In particular, the lowest, most important surface mode frequency is easily calculable for a small ellipsoidal particle, as mentioned in Sect. 2.

The quantity to be fitted now, by a superposition of Lorentztype profiles, is the extinction cross section per unit volume $C_{\text {ext }}(\omega) / V$ taken from our transmission measurements and expected to depend explicitly on the shape of the grains. 
The extinction cross section per unit volume averaged over all orientations, and over all shape distributions of ellipsoidal grains with the volume $V$, takes the well known form (Bohren \& Huffman 1983):

$<<C_{\text {ext }}(\omega)>>/ V=\frac{1}{3} k \operatorname{Im} \sum_{i=1}^{3} I_{i}(\omega)$,

where the summation goes over the three main semi-axes of the ellipsoids and $k$ denotes the length of the wave vector, while:

$I_{i}(\omega)=\iint P\left(L_{1}, L_{2}\right) \frac{\epsilon(\omega)-1}{1+L_{i}(\epsilon(\omega)-1)} \mathrm{d} L_{1} \mathrm{~d} L_{2}$.

The shape probability function $P\left(L_{1}, L_{2}\right)$ defines the relative weight of the geometrical factors $L_{i}$ over the integration range $\left\{0<L_{1}<1,0<L_{2}<1-L_{1}\right\}$ with $L_{1}+L_{2}+L_{3}=1$. Alternatively, it determines the probability of finding, in the collection, the ellipsoids with given ratios of lengths of the three semi-axes. In general then, the choice of a particular distribution could be either motivated a priori by e.g. a microscopic analysis of the sample or justified a posteriori by the quality of the fit.

Various shape distributions have been proposed in the past for the just defined continuous distribution of ellipsoids. Two of them have been taken into consideration in the present study. The first, hereinafter named "flat CDE", is defined by the condition $P\left(L_{1}, L_{2}\right)=2$, meaning that this distribution does not favour any particular shape, with the consequence that e.g. spheres and needles are equally probable. It was used by Bohren \& Huffman (1983) to explain the IR extinction spectra of quartz, $\mathrm{SiC}$ and $\mathrm{MgO}$ particles. The second, hereinafter named "peaked CDE", obtained by assuming $P\left(L_{1}, L_{2}\right)=$ $120 L_{1} L_{2} L_{3}$, is maximum at $L_{i}=1 / 3$ and vanishes at $L_{i}=0$ $(i=1,2,3)$. It favours therefore the almost spherical particles and minimises the contribution of disks and needles. Ossenkopf et al. (1992) used such a distribution to retrieve the optical constants of interstellar silicate grains.

The task is now to fit our experimental data by a properly chosen set of Lorentzian profiles by using Eqs. (4) and (5) with:

$\epsilon(\omega)=\epsilon_{\infty}+\sum_{j} \frac{F_{j}}{\omega_{j}^{2}-\omega^{2}-i \gamma_{j} \omega}$

where $F_{j}$ denotes the so-called line strength of the $j$ th oscillator, $\omega_{j}$ its $j$ th eigenfrequency and $\gamma_{j}$ its damping factor.

Finally, one can derive the refractive index of the grain material, $m=n+i k$ from the dielectric constant $\epsilon$ using the relation $m=\sqrt{\epsilon}$ (where the dependence on frequency $\omega$ is implicit).

The retrieved values of the spectral refractive index are then compared with those obtained for spherical particles.

For a collection of such particles, $P\left(L_{1}, L_{2}\right)=\delta\left(L_{1}-\right.$ $\left.\frac{1}{3}\right) \delta\left(L_{2}-\frac{1}{3}\right)$, hence

$$
\begin{aligned}
I_{i}(\omega) & =\iint \delta\left(L_{1}-\frac{1}{3}\right) \delta\left(L_{2}-\frac{1}{3}\right) \frac{\epsilon(\omega)-1}{1+L_{i}(\epsilon(\omega)-1)} \mathrm{d} L_{1} \mathrm{~d} L_{2} \\
& =3 \frac{\epsilon(\omega)-1}{\epsilon(\omega)+2} .
\end{aligned}
$$

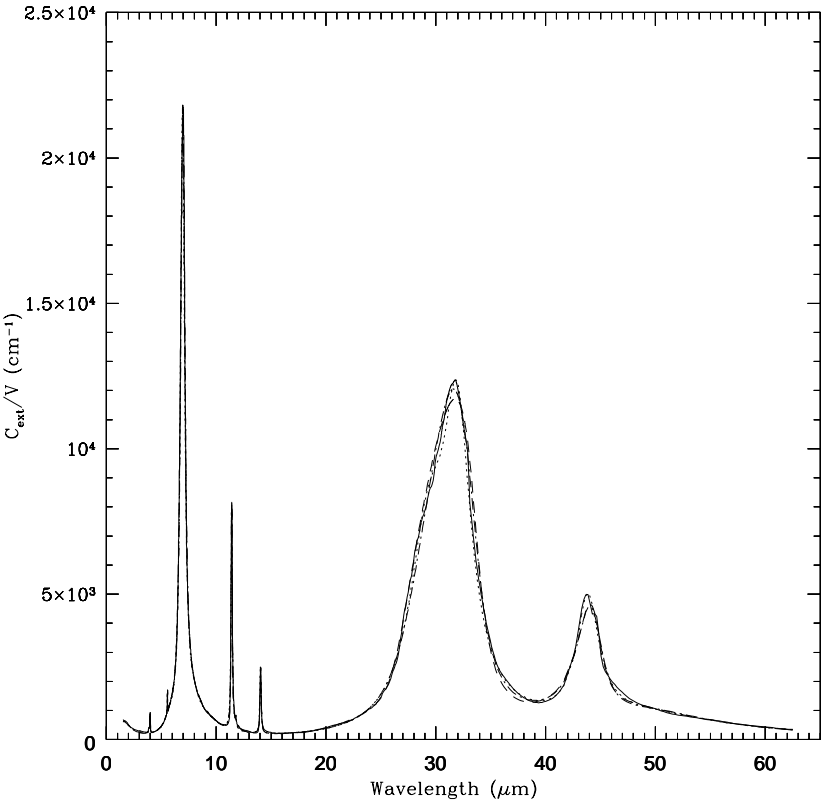

Fig. 2. Best fits to the experimental extinction spectrum of limestone particles (solid curve) obtained in this work for the flat CDE (dashed curve) and the peaked CDE (dot-dashed curve) and by Orofino et al. (2002) for spherical grains (dotted curve). Seventeen Lorentz oscillators have been used to carry out the fits to the three different distributions.

Then

$$
\begin{aligned}
<<C_{\text {ext }}(\omega)>>/ V & =\frac{1}{3} k \operatorname{Im} \sum_{i=1}^{3} I_{i}(\omega) \\
& =3 k \operatorname{Im} \frac{\epsilon(\omega)-1}{\epsilon(\omega)+2}
\end{aligned}
$$

as presented in the paper by Orofino et al. (1998).

The IR transmission spectra of our limestone particles have been obtained in the wavelength range $1.5-62.5 \mu \mathrm{m}$ by means of the usual pellet technique, using both Potassium Bromide (KBr) and Cesium Iodide (CsI) as transparent matrices in which limestone grains have been dispersed. The experimental procedure has been described in more details in a previous work (Orofino et al. 2002).

\section{Results and conclusions}

The approach outlined in Sect. 3 has been applied first to the flat CDE. As initial input parameters for the fitting procedure, we have used the best-fit values for the 17 contributing Lorentzian oscillators obtained by Orofino et al. (2002), by assuming the spherical shape of the grains. The minimization was very fast: even if the fitting procedure typically converged after 90 iterations, the CPU time was only of a few seconds. The resulting best-fit parameters have been then used as input data for the peaked CDE. Also in this case the minimization was very fast (convergence reached after 26 iterations), producing a new set of best-fit parameters.

Figure 2 reports the best fits to the experimental extinction spectrum of our limestone particles obtained for the two shape distributions (flat and peaked CDE) over the whole spectral 


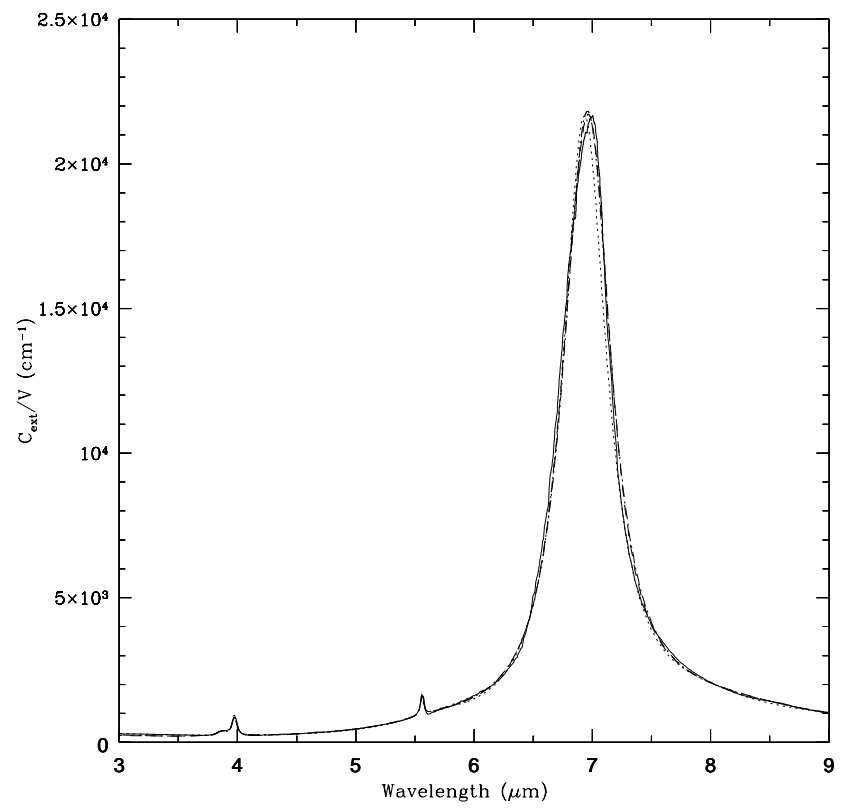

Fig. 3. Same as in Fig. 2 but for the particular spectral range $3-9 \mu \mathrm{m}$.

range under consideration. In the same figure it is also shown the best fit obtained for spheres with the same number of oscillators (Orofino et al. 2002). In Figs. 3-5 are shown, additionally, the details of the best fits in three separate spectral ranges: 3-9 $\mu \mathrm{m}$ (Fig. 3), 10-16 $\mu \mathrm{m}$ (Fig. 4), and 20-50 $\mu \mathrm{m}$ (Fig. 5). As it can be seen, up to $20 \mu \mathrm{m}$ the fits, obtained with the three shape distributions, almost overlap. Some remarkable discrepancies appear, on the contrary, beyond $20 \mu \mathrm{m}$. In order to understand the reasons of such differences it is important to note that the third spectral range is dominated by lattice surface modes. In particular the two bands shown up by our limestone samples at about $32 \mu \mathrm{m}$ and $44 \mu \mathrm{m}$ are very close to the so-called $v_{6}$ and $v_{9}$ lattice modes of the $\mathrm{CaCO}_{3}$ structure (White 1974). It is therefore obvious that the effects of the different shape distributions of the grains are evident mostly in the region of these bands.

For all the three shape distributions the fits are quite good and the chi-squared values are very close to each other. If, however, we consider the ability of reproducing the height of the bands as a criterion of the quality of the fit, then the best fit is obtained for spheres, while for peaked CDE and for flat CDE the fits become increasingly worse (see Fig. 5). This may indicate the relevance of quasi-spherical particles in the specimen. Such a conclusion is in agreement with SEM images of our samples (Fig. 1), where it is possible to note that most of the isolated grains are approximately spheroidal in shape. The SEM image shows also the presence of clusters, composed of smaller particles, having more irregular shapes. However, during the pellet production needed for the IR spectroscopy, these clusters are disaggregated, resulting in isolated grains dispersed over the matrix.

Figure 6 reports the spectral dependence of the three sets of optical constants derived from the best fits to the experimental spectrum and obtained with the three different shape distributions. For the sake of the forthcoming discussion the optical

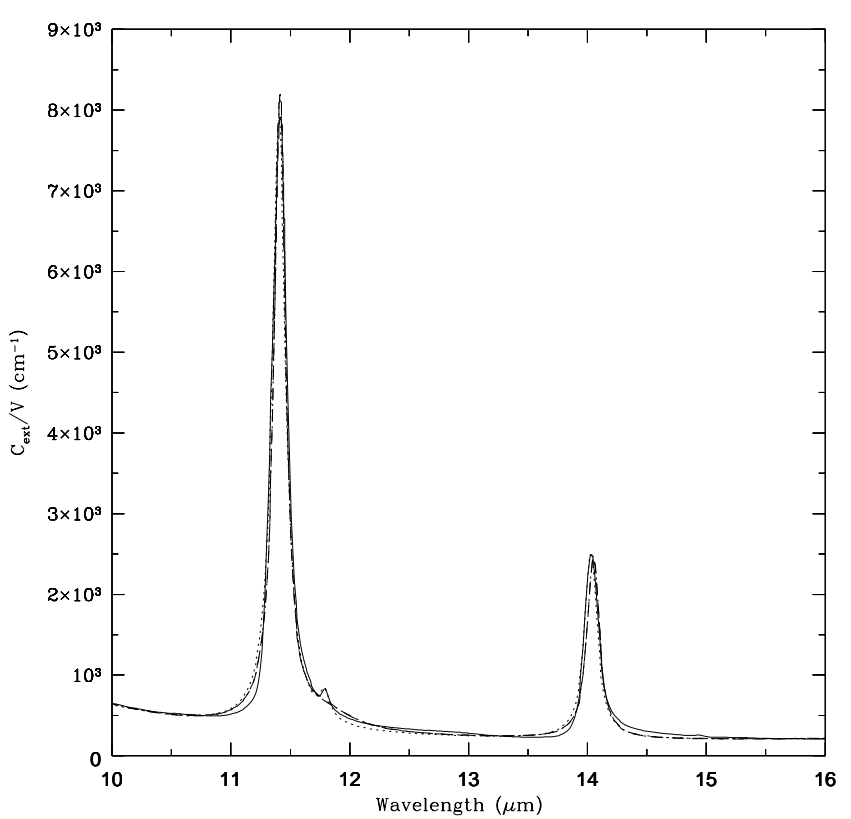

Fig. 4. Same as in Fig. 2 but for the particular spectral range 10-16 $\mu \mathrm{m}$.

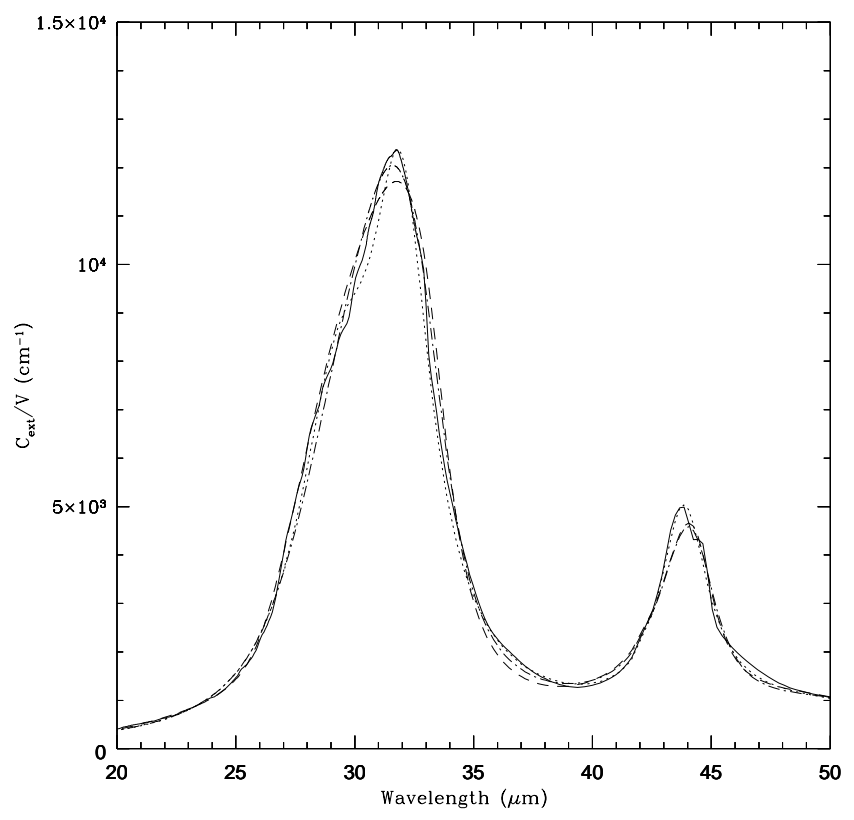

Fig. 5. Same as in Fig. 2 but for the particular spectral range $20-50 \mu \mathrm{m}$.

constants, derived by Long et al. (1993) for pressed pellets of calcite grains, are also shown.

It is difficult to assess the errors on both the real and the imaginary parts of the refractive index. Actually, they can be derived from a fitting procedure able to reproduce the measured extinction efficiencies within their relative experimental errors. These have usually two main causes: a) the uncertainty on the total mass of the embedded particles and b) the differences in the spectral behaviour of the embedding matrices relative to different samples. Since these experimental errors, depending on the wavelength range, affect to different extent the uncertainty on $n$ and $k$, it is practically impossible to determine exactly these uncertainties. However, the errors on $n$ and $k$ are of 

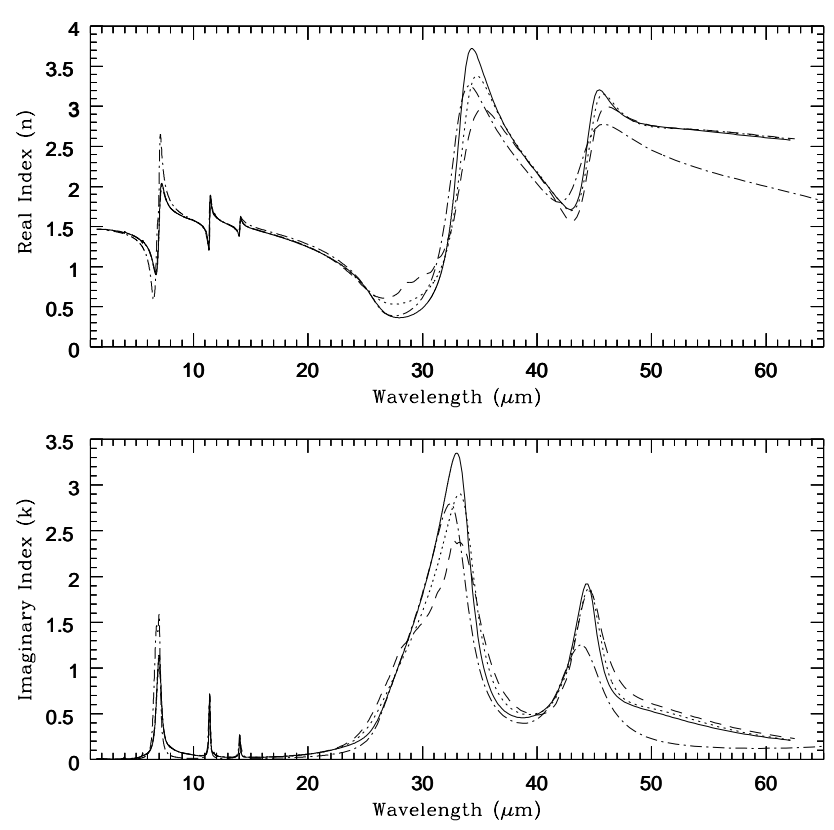

Fig. 6. Real (top panel) and imaginary (bottom panel) part of the refractive index of particulate limestone samples obtained in this work for the flat (solid curve) and the peaked CDE (dotted curve), compared with those calculated for spheres (dashed curve) by Orofino et al. (2002) and for the semi-bulk sample (dot-dashed curve) by Long et al. (1993).

the order of a few percent within the band regions and something more in the continuum.

Figure 6 clearly shows that, starting from the same experimental extinction spectrum, it is possible in general to obtain different sets of optical constants depending on the adopted shape distribution. In particular the three sets of particulate optical constants shown in Fig. 6 are almost the same in the short-wavelength range, while they are markedly distinct in the long-wavelength range. The discrepancies in the retrieved optical constants are even stronger than the differences among our three fits presented in Figs. 2-5. This pertains especially to the bands at about $32 \mu \mathrm{m}$ and $44 \mu \mathrm{m}$ which originate from lattice surface modes. The most important differences in both $n$ and $k$ obtained for the three shape distributions appear around $32 \mu \mathrm{m}$ and concern both the profile and position of the band. We recall that, while in the peaked CDE the spherical shape is privileged, in the flat $\mathrm{CDE}$ all shapes are equally distributed. In other words, of the two CDE distributions, the flat one is "more different" from pure spheres than the peaked. The optical constants obtained for spherical grains and those for particles with flat CDE show up, for this reason, the major discrepancies. These differences strongly indicate the importance of an adequate treatment of the boundary conditions in the Maxwell theory of interaction between radiation and matter. Both the SEM analysis and the best fit of the experimental extinction spectrum would give us the best shape distribution among the shape distributions used in the fitting procedure, and, as a consequence, the best set of optical constants. In our case the best optical constants are those derived by using Mie theory, since both SEM analysis and best fit seem to indicate a spheroidal shape of our limestone particles (see discussion above).
The similarity of the spectral behaviour of the three sets of optical constants at the short-wavelength range indicates that in those spectral regions, where surface modes are not effective, the shape distribution is not relevant to the derivation of optical constants. For this reason Mie theory is sufficient to this goal.

In Fig. 6 it is also shown the comparison between our particulate optical constants and those obtained for the same material by Long et al. (1993). These authors obtained their data from reflection measurements on pressed pellets of randomly oriented calcite grains. This experimental method produces optical constants intermediate between bulk and particulate ones, as already pointed out in an earlier work (Orofino et al. 1998). Figure 6 clearly indicates what was previously found for the case of spheres (Orofino et al. 1998, 2002), namely that the optical constants of particulate samples of limestone are different from those obtained by Long et al. (1993) for their "semi-bulk" samples. In the present study the most important differences with respect to semi-bulk optical constants appear for particles with flat CDE. These discrepancies are remarkable both in $n$ and $k$ for the two bands around 32 and $44 \mu \mathrm{m}$. Unfortunately, a similar comparison cannot be carried through the optical constants obtained by Querry et al. (1978) for bulk samples of limestone. This is because these quantities, derived from reflection measurements for polished pieces of rock having more than $99.7 \%$ of calcite, are given in the spectral range $0.2-32.8 \mu \mathrm{m}$. However, on the basis of the results obtained in the same spectral region by Orofino et al. (1998), we can expect that the discrepancies between our particulate optical constants and those pertaining bulk limestone should be even more pronounced than the differences existing between particulate and semi-bulk quantities.

The results presented here have strong implications for the calculations of synthetic spectra of Mars, whenever particles smaller than the wavelength are involved in radiative processes. This pertains to transmission spectra of airborne particles (aerosol) present in the Martian atmosphere as well as to emission spectra of the smallest grains present in the regolith of some bright areas of Mars. In fact nearly all the aerosol grains have sizes smaller than $30 \mu \mathrm{m}$ (Toon et al. 1977; Clancy et al. 1995), with an average effective radius ranging from $0.2 \mu \mathrm{m}$ (Chylek \& Grams 1978) to $2.75 \mu \mathrm{m}$ (Toon et al. 1977) depending on the epoch of observation, the location of the observed area on Mars and the assumed dust composition (see Pollack et al. 1995 for a review on the subject). Also, the thermal inertia of the regolith present in some areas of the Martian surface, such as Tharsis, Arabia and Elysium, has been recently found to be less than $60 \mathrm{~J} \mathrm{~m}^{-2} \mathrm{~s}^{-0.5} \mathrm{~K}^{-1}$ (Jakosky et al. 2000; Mellon et al. 2000), that corresponds to particle sizes smaller than $1 \mu \mathrm{m}$ (Presley \& Christensen 1997).

For such kinds of particles bulk optical constants have until now been generally used in order to produce synthetic spectra of Mars. As found by Orofino et al. (1998), this approach could lead, however, to wrong results regarding in particular the band intensities and therefore the abundance of carbonate materials expected on Mars. Such results strongly suggested that for a reliable evaluation of the Martian spectra produced by small grains, particulate optical constants are necessary. The new data presented here confirm those previous and clarify 
the possible spectral influence of the size and shape of limestone grains on their transmission and/or emission spectra. At short wavelengths, synthetic Martian spectra for small limestone particles can be calculated by using particulate optical constants derived for spheres by Mie approximation, independently of the real shape distribution of the Martian dust. On the other hand, however, in the spectral region where surface modes appear, for materials like limestone, the synthetic spectra have to be calculated using the optical constants derived by taking into account the real shapes and sizes of the particles. Finally, it is worthwhile to note that the conclusion reached here for limestone particles cannot be extrapolated directly to other particles and/or materials. Indeed, every case should be treated independently.

Acknowledgements. The authors warmly thank A. Perrone and T. Roush for useful discussions and M. D'Elia for SEM analysis. This research was supported by the Polish State Committee for Scientific Research under grant No. 2P03C 00217 and partially by the Ministry of Public Education and Research (MIUR) and the Italian Space Agency (ASI).

\section{References}

Albee, A. L., Arvidson, R. E., Palluconi, F., \& Thorpe, T. 2001, J. Geophys. Res., 106, 23291

Baker, V. R., Strom, R. G., Gulick, V. C., Kargel, J. S., \& Komatsu, G. 1991, Nature, 352, 589

Bohren, C. E., \& Huffman, D. R. 1983, Absorption and scattering of light by small particles (New York: Wiley)

Born, M., \& Huang, K. 1954, Dynamical Theory of Crystal Lattices (Oxford: Clarendon)

Bryksin, V. V., Gerbshtein, Yu. M., \& Mirlin, D. N. 1971, Sov. Phys. Solid State, 13, 1342

Bryksin, V. V., Mirlin, D. N., \& Firsov, Yu. A. 1974, Sov. Phys. Usp., 17,305

Cabrol, N. A., \& Grin, E. A. 1999, Icarus, 142, 160

Carr, M. H. 1999, J. Geophys. Res., 104, 21897

Chen, T. S., de Wette, F. W., \& Kleinman, L. 1978, Phys. Rev. B, 18, 958

Chylek, P., \& Grams, G. W. 1978, Icarus, 36, 198

Clancy, R. T., Lee, S. W., Gladstone, G. R., McMillan, W. W., \& Roush, T. 1995, J. Geophys. Res., 100, 5251

Conel, J. E. 1969, J. Geophys. Res., 74, 1614

Duley, W. W. 1976, Ap\&SS, 39, L33

Englman, R., \& Ruppin, R. 1966, Phys. Rev. Lett., 16, 898

Englman, R., \& Ruppin, R. 1968, J. Phys. C, 1, 614

Fonti, S., Jurewicz, A., Blanco, A., Blecka, M. I., \& Orofino, V. 2001, J. Geophys. Res., 106, 27815

Forget, F., \& Pierrehumbert, R. T. 1997, Science, 278, 1273

Fröhlich, H. 1949, Theory of Dielectrics (London: Oxford)

Fuchs, R. 1973, Phys. Lett. A, 43, 42

Fuchs, R. 1975, Phys. Rev. B, 11, 1732

Fuchs, R. 1978, Phys. Rev. B, 18, 7160

Fuchs, R. 1987, Phys. Rev. B, 35, 7700

Fuchs, R., \& Kliewer, K. L. 1968, J. Opt. Soc. Am., 58, 319

Fuchs, R., Kliewer, K. L., \& Pardee, W. J. 1966, Phys. Rev., 150, 589

Genzel, L., \& Martin, T. P. 1972a, Phys. Stat. Solid B, 51, 91

Genzel, L., \& Martin, T. P. 1972b, Phys. Stat. Solid B, 51, 101
Golombek, M. P., Cook, R. A., Economou, T., et al. 1997, Science, 278,1743

Grindlay, J. 1965, Can. J. Phys., 43, 1604

Haberle, R. M. 1998, J. Geophys. Res., 103, 28467

Huang, K. 1951, Proc. Roy. Soc. A, 208, 352

Huffman, D. R. 1977, Adv. Phys., 26, 129

Huffman, D. R. 1988, in Experiments on Cosmic Dust Analogues, ed. E. Bussoletti et al. (Dordrecht: Kluwer Academic), 25

Hunt, G. R., \& Logan, L. M. 1972, Appl. Opt., 11, 142

Jakosky, B. M., Mellon, M. T., Kieffer, H. H., et al. 2000, J. Geophys. Res., 102, 9643

Jurewicz, A., Blecka, M. I., Blanco, A., Fonti, S., \& Orofino, V. 2001, Adv. Space Res., 28, 1191

Landolt-Börnstein 1955, Zahlenwerte und Funktionen, 6 Aufl. Bd. I/4 (Berlin, Göttingen, Heidelberg: Springer), 604

Long, L. L., Querry, M. R., Bell, J. R., \& Alexander, R. W. 1993, Infrared Phys., 34, 191

Lyddane, R. H., Sachs, R. G., \& Teller, E. 1941, Phys. Rev., 59, 673

Malin, M. C., \& Carr, M. H. 1999, Nature, 397, 589

Martin, T. P. 1975, Phys. Rev. B, 7, 3907

Masursky, H., Boyce, J. M., Dial, A. L., Schaber, G. G., \& Strobell, M. E. 1977, J. Geophys. Res., 82, 4016

McKay, D. S., Gibson, E. K., Thomas-Keprta, K. L., et al. 1996, Science, 273, 924

Mellon, M. T., Jakosky, B. M., Kieffer, H. H., \& Christensen, P. R. 2000, Icarus, 148, 437

Morse, P., \& Feshbach, H. 1953, Methods of Theoretical Physics (New York: McGraw Hill)

Muci, A. M., Blanco, A., Fonti, S., \& Orofino, V. 1997, Adv. Space Res., 20, 1605

Orofino, V., Blanco, A., Fonti, S., Proce, R., \& Rotundi, A. 1998, Planet. Space Sci., 46, 1659

Orofino, V., Blanco, A., Blecka, M. I., Fonti, S., \& Jurewicz, A. 2000, Planet. Space Sci., 48, 1341

Orofino, V., Blanco, A., Fonti, S., Marra, A. C., \& Polimeno, N. 2002, Planet. Space Sci., 50, 839

Ossenkopf, V., Henning, Th., \& Mathis, J. S. 1992, A\&A, 261, 567

Pollack, J. B., Kasting, J. F., Richarsson, S. M., \& Poliakoff, K. 1987, Icarus, 71, 203

Pollack, J. B., Ockert-Bell, M. E., \& Shepard, M. K. 1995, J. Geophys. Res., 100, 5235

Presley, M. A., \& Christensen, P. R. 1997, J. Geophys. Res., 102, 6551

Querry, M. R., Osborne, G., Lies, K., Jordon, R., \& Coveney, Jr. R. M. 1978, Appl. Opt., 17, 353

Rouleau, F., \& Martin, P. G. 1991, ApJ, 377, 526

Roush, T., Pollack, J. B., \& Orenberg, J. 1991, Icarus, 94, 191

Ruppin, R., \& Englman, R. 1968, J. Phys. C, 1, 630

Ruppin, R., \& Englman, R. 1970, Rep. Progr. Phys., 33, 149

Sawyer, D. J., McGehee, M. D., Canepa, J., \& Moore, C. B. 2000, Meteorit. Planet. Sci., 35, 743

Scott, D. H., Dohm, J. M., \& Rice, J. W. Jr. 1995, U.S. Geological Survey, Misc. Invest. Series, Map I-2461

Thomas-Keprta, K. L., Bazylinski, D. A., Kirschvink, J. L., et al. 2000, Geochim. Cosmochim. Acta, 64, 4049

Toon, O. B., Pollack, J. B., \& Sagan, C. 1977, Icarus, 30, 663

White, W. B. 1974, The carbonate minerals in The Infrared Spectra of Minerals, ed. V. C. Farmer (Mineralogical Society of London), 227

Yung, Y. L., Nair, H., \& Gerstell, M. F. 1997, Icarus, 130, 222

Zubko, V. G., Mennella, V., Colangeli, L., \& Bussoletti, E. 1996, MNRAS, 282, 1321 